\title{
Role of matrix metalloproteinases and their tissue inhibitors in the pathological mechanisms underlying maxillofacial cystic lesions
}

\author{
KRYSTIAN KUŹNIARZ ${ }^{1}$, DOROTA LUCHOWSKA-KOCOT ${ }^{2}$, TOMASZ TOMASZEWSKI $^{1}$ and JACEK KURZEPA ${ }^{2}$ \\ ${ }^{1}$ Department of Maxillofacial Surgery; ${ }^{2}$ Department of Medical Chemistry, \\ Medical University of Lublin, Lublin 20-081, Poland
}

Received February 17, 2021; Accepted April 27, 2021

DOI: $10.3892 /$ br.2021.1441

\begin{abstract}
Cystic lesions are considered to be one of the most common pathologies of the maxillofacial region, and matrix metalloproteinases (MMPs) may represent potential etiological factors. The aim of the present study was to elucidate the role of MMP-2 and MMP-9, and their endogenous tissue inhibitors, tissue inhibitor of metalloproteinase (TIMP)-1 and TIMP-2, respectively, in the pathogenesis of maxillofacial cystic lesions. A total of 25 patients diagnosed with radicular cysts (RCs; $n=20)$, dentigerous cysts $(n=3)$ and retention cysts (RtCs; $n=7$ ) were enrolled in the present study. Gelatin zymography was performed to assess the gelatinolytic activity of MMP-2 and MMP-9, and commercial ELISA kits were used to determine TIMP-1 and TIMP-2 concentrations. Gelatin zymography revealed the presence of both MMP-2 and MMP-9 in all types of samples analyzed. An increase in MMP-9 activity, TIMP-1 concentration and MMP-9/TIMP-1 ratio was observed in the fluid obtained from RCs compared with that obtained from RtCs. In conclusion, MMP-9 may be involved in the pathogenesis of RCs, whereas the activity of MMP-2 in the wall of RtCs was low, and this gelatinase did not appear to significantly affect the development of this type of lesion.
\end{abstract}

\section{Introduction}

Cystic lesions are amongst the most common pathologies diagnosed in the maxillofacial region. They may occur in facial soft tissues as well as the craniofacial skeleton. Moreover, these anomalies are characterized by distinct etiopathogenetic processes (1-3). The most frequent cystic lesions localized within the jaws are radicular cysts (RCs), which are associated with the development of periapical granulomas (PGs) that form as a result of untreated periapical periodontitis (4). The

Correspondence to: Dr Krystian Kuźniarz, Department of Maxillofacial Surgery, Medical University of Lublin, 11 Staszica, Lublin 20-081, Poland

E-mail:krystiankuzniarz@umlub.pl

Key words: odontogenic cyst, radicular cyst, dentigerous cyst, retentions cyst, matrix metalloproteinase, tissue inhibitor of metalloproteinase, zymography, matrixins second most prevalent type of odontogenic cystic lesion, the dentigerous cyst (DC), is characterized as a developmental cyst (5). Despite the distinct differences in the etiology of the respective pathological lesions, their growth processes are predominantly associated with resorption and remodeling of bone tissue (6). Numerous studies have been conducted to investigate the mechanisms involved in both types of lesions. MMPs are a group of proteins potentially involved in cyst formation and bone destruction (7-11). This family of zinc-dependent enzymes is responsible for the degradation of the extracellular matrix (ECM) during various physiological and pathological processes, including in embryogenesis, organization of dentin organic matrix, arthritis and ischemic stroke $(12,13)$. Moreover, proteolytic activity of these endopeptidases is observed in angiogenesis during wound healing and cancer metastasis $(14,15)$. Tissue inhibitors of metalloproteinases (TIMPs) comprise a group of specific endogenous enzymes regulating the activity of MMPs. Thus, TIMPs serve a key role in the maintenance of homeostasis and proper functioning of the body as a whole (16).

MMP-2 and MMP-9 belong to a well-known subgroup of MMPs termed gelatinases $(11,17)$. Since some of bone organic components (for example, type I collagen and fibronectin) are substrates for these endopeptidases, the possible involvement of MMP-2 (also known as gelatinase A) and MMP-9 (also known as gelatinase B) in the etiology of cystic lesions has been investigated $(18,19)$. Accordingly, cleavage of the organic elements of the ECM in bone tissue may lead to cyst formation, and gene expression analysis revealed higher expression of MMP-2, TIMP-1 and TIMP-2 in PGs and RCs, underlining its significant role in the etiology of these disorders (20). Ameloblastomas, which are considered as more expansive pathologies than benign lesions, are also characterized by higher expression of MMPs (21). However, the etiopathogenesis of DCs and MMPs remains unclear (22). Retention cysts (RtCs) are lesions occurring within the maxillary sinuses and exhibit a lower destructive potential compared with typical odontogenic cysts (23). Unfortunately, our knowledge regarding the involvement of MMPs in the etiology of RtCs is limited. Searching PubMed with the phrase 'retention cysts' and 'MMP' returned only 1 article (24). Furthermore, to the best of our knowledge, there are currently no studies comparing MMP activity and TIMP levels between RtCs and the aforementioned odontogenic cysts. 
Therefore, the aim of the present study was to understand and characterize the role of MMP-2, MMP-9 and their tissue inhibitors, TIMP-1 and TIMP-2, in the pathogenesis of cystic lesions of the maxillofacial region.

\section{Materials and methods}

Patients. A total of 25 patients (16 males and 9 females), aged 18-66 years old (median age, 43.2), who were diagnosed with cystic lesions of the maxillofacial region were enrolled in the present study. In the investigated group of patients, $20 \mathrm{RCs}$, 7 RtCs and 3 DCs were identified using clinical examination, computed tomography and/or panoramic X-ray imaging. The higher number of cysts detected than patients recruited was due to the presence of multiple comorbidities amongst patients. In addition, 2 separate RCs were identified in each patient of a group of 3 patients; 1 patient was diagnosed with 2 RCs and $1 \mathrm{RtC}$; and $1 \mathrm{RC}$ and $1 \mathrm{RtC}$ were identified in 1 patient. The characteristics of all the lesions were confirmed by preliminary histopathological examination. Studies were performed using cystic tissues collected during surgical treatment conducted at the Department of Maxillofacial Surgery, Medical University of Lublin. A total of 2 lesions with inconsistencies between the clinical, histopathological or radiological diagnoses were excluded from the study. Moreover, due to the non-specific microscopic characteristics of RtCs, concomitant histopathological, clinical and radiological examinations were considered during evaluation for inclusion in the study (25).

All study protocols were reviewed and approved by the Ethics Committee at the Medical University of Lublin (approval no. KE-0254/5/2017). Each patient included in the study provided signed informed consent. All procedures followed the guidelines outlined in the Declaration of Helsinki (26). The characteristics of the study group are summarized in Table I.

Sample collection and preparation. A sample of venous blood was routinely collected prior to surgery under general anesthesia. The blood was centrifuged at 1,800 x g for $20 \mathrm{~min}$ at room temperature and $0.5-1.0 \mathrm{ml}$ of the obtained serum was collected in Eppendorf tubes and stored at $-30^{\circ} \mathrm{C}$. The remaining serum was used to perform biochemical blood tests. Cystic lesions were exposed during surgery, and cystic fluid was obtained by diagnostic puncture and aspiration from the cavity using a sterile syringe. Subsequently, enucleation of the entire cyst capsule was performed. Finally, a small fragment (0.3-0.5 g) was detached from the capsule using a sterile surgical blade. All cyst tissue samples were placed in separate Eppendorf tubes and frozen at $-30^{\circ} \mathrm{C}$. The primary part of the lesion was subjected to histopathological examination at the Pathomorphology Unit of the Independent Public Teaching Hospital No. 1 to confirm the initial diagnosis. All subsequent tests were performed at the Department of Medical Chemistry, Medical University of Lublin.

The samples detached from the capsules were homogenized using buffer containing 1\% Triton X-100 and centrifuged at $1,800 \mathrm{x} \mathrm{g}$ for $20 \mathrm{~min}$ at room temperature. Protein levels in all supernatant samples were determined using commercial Bradford reagent (Bio-Rad Laboratories, Inc.) according to manufacturer's protocol $(18 \mu \mathrm{g} / \mathrm{ml})$. In each examined case, serum and cyst fluid samples were diluted (1:50) with deionized water.
Table I. Characteristics of the study group.

\begin{tabular}{lccc}
\hline Characteristics & $\begin{array}{c}\mathrm{RC}, \\
\mathrm{n}=20\end{array}$ & $\begin{array}{c}\mathrm{RtC}, \\
\mathrm{n}=7\end{array}$ & $\begin{array}{c}\mathrm{DC}, \\
\mathrm{n}=3\end{array}$ \\
\hline Average age, years & 42.4 & 41.6 & 44.3 \\
Sex, male/female & $12 / 5$ & $4 / 3$ & $1 / 2$ \\
Localization, maxilla/mandible & $16 / 4$ & - & $1 / 2$ \\
Maxillary sinus, right/left & - & $2 / 5$ & - \\
Average largest dimension, mm & 21.8 & 27.2 & 14.3 \\
\hline
\end{tabular}

$\mathrm{RC}$, radicular cyst; RtC, retention cyst; DC, dentigerous cyst; $\mathrm{n}$, number of examined lesions.

Zymography. All zymographic procedures (incubation, protein separation, staining/distaining) were performed at room temperature. The proteolytic activity of MMP-2 and MMP-9 in the serum, cyst fluid and cyst capsules were detected and evaluated using gelatin zymography (27). Initially, $30 \mu \mathrm{l}$ study material was mixed with $10 \mu \mathrm{l}$ sample buffer with $10 \%$ SDS and incubated for $30 \mathrm{~min}$. Subsequently, $20 \mu \mathrm{l}$ of this solution was loaded on a $10 \%$ SDS-gel with $0.05 \%$ gelatine type A from porcine skin (Sigma-Aldrich; Merck $\mathrm{KGaA}$ ), and resolved using SDS-PAGE for $90 \mathrm{~min}$ at $30 \mathrm{~mA} / \mathrm{gel}$ at room temperature. Next, the resolved proteins were incubated with Tris- $\mathrm{HCl}$ buffer $(\mathrm{pH}$ 7.2) containing $10 \mathrm{mM}$ $\mathrm{CaCl}_{2}, 0.02 \% \mathrm{NaN}_{3}$ and $2.5 \%$ Triton X-100 twice for $30 \mathrm{~min}$. Subsequent incubation was performed for $18 \mathrm{~h}$ at $37^{\circ} \mathrm{C}$ using Tris-HCl buffer ( $\mathrm{pH}$ 7.2) containing $10 \mathrm{mM} \mathrm{CaCl}_{2}, 0.02 \% \mathrm{NaN}_{3}$ and $1 \%$ Triton X-100. Gels were stained with $0.1 \%$ Coomassie Blue R-250 in solution of $30 \%$ ethanol and $10 \%$ acetic acid for $4 \mathrm{~h}$. Finally, they were destained twice in solution of $30 \%$ ethanol and $10 \%$ acetic acid. The emergence of clear bands against the blue background indicated the activity of MMP-2 and MMP-9 (28). Investigation using gelatine zymography was performed simultaneously on two separate gels to avoid inconclusive results.

Enzymes were identified by comparing their localization with molecular mass standards (SM0441; Fermentas; Thermo Fisher Scientific, Inc.) and with standards of both gelatinases (911-MP and 902-MP; R\&D Systems, Inc.). Quantitative analysis of MMP activity was performed using a computer scanner (1,200 dpi) and ImageJ 1.51.9 (National Institutes of Health). The activity of the tested enzymes was expressed as the optical density (OD) of the substrate lysis zone.

ELISA. TIMP-1 and TIMP-2 levels were detected in serum and cyst fluid using commercially available ELISA kits (cat. nos. DMP100 and DMP200; R\&D Systems Inc.) according to the manufacturer's protocol. Both tissue inhibitors were diluted 100 times with the diluent included in the kit. All results were detected using a microplate reader (Epoch; BioTek Instruments, Inc.) at a wavelength of $450 \mathrm{~nm}$. Moreover, MMP-9/TIMP-1 and MMP-2/TIMP-2 ratios, as calculated from zymography and ELISA results, were expressed in OD/ng/ml.

Statistical analysis. A Kruskal-Wallis test followed by a Dunns' post hoc test was used for statistical analysis. Data are presented as the median and the interquartile range. Statistical analysis was performed using InStat version 3.06 (GraphPad 


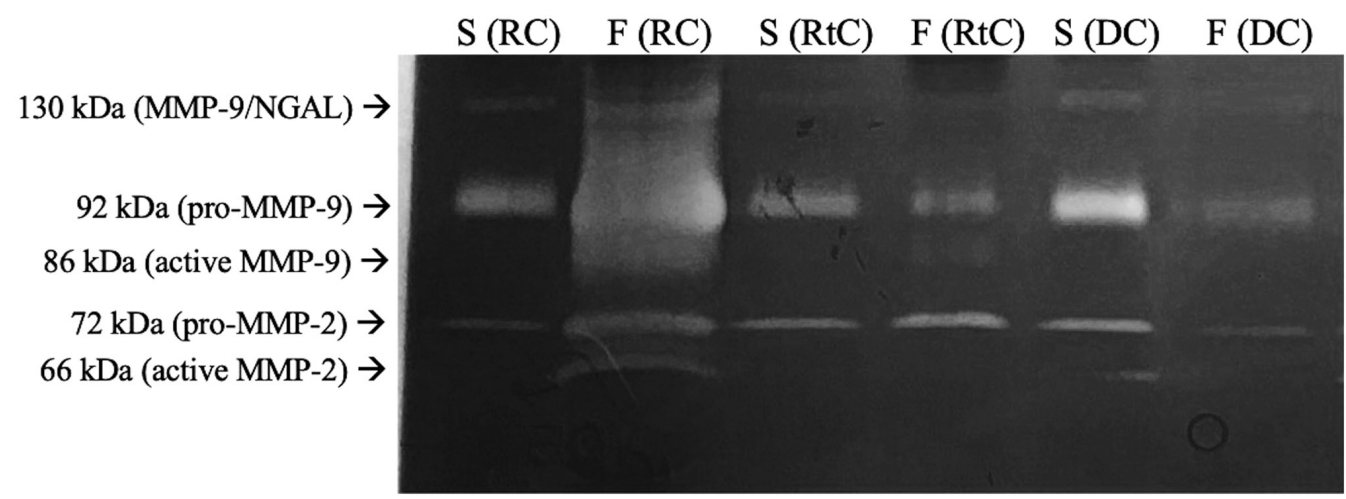

Figure 1. Representative zymogram of the serum and cyst fluid from patients with RC, DC or RtC. S, serum; F, fluid; RC, radicular cyst; RtC, retention cyst; DC, dentigerous cyst; MMP, matrix metalloproteinase; NGAL, neutrophil gelatinase-associated lipocalin.

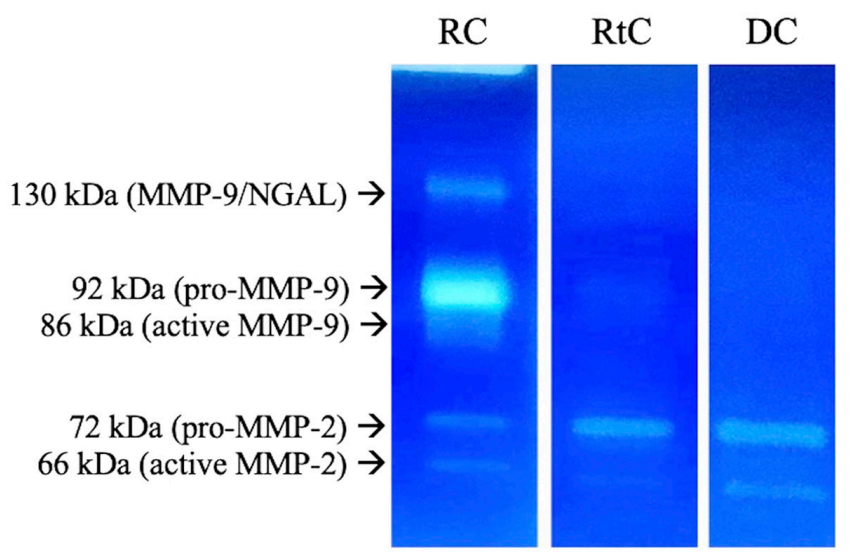

Figure 2. Representative zymogram of the cyst capsules of patients with RC, DC or RtC. RC, radicular cyst; RtC, retention cyst; DC, dentigerous cyst; MMP, matrix metalloproteinase.

Software, Inc.). $\mathrm{P}<0.05$ was considered to indicate a statistically significant difference.

\section{Results}

Zymography analysis confirmed the activity of MMP-2 and MMP-9 in all samples analyzed. A representative zymogram showing the proteolytic activity of both gelatinases in the serum and cyst fluid of patients with RCs, DCs and RtCs is presented in Fig. 1. Gelatinolytic activity was detected at molecular weights of 66 and $86 \mathrm{kDa}$, corresponding to the active forms of MMP-2 and MMP-9, respectively. Additionally, a $92 \mathrm{kDa}$ band was present, which corresponds to the latent form of MMP-9 (pro-MMP-9), and the $72 \mathrm{kDa}$ band was associated with pro-MMP-2. Moreover, an MMP-9/neutrophil gelatinase-associated lipocalin heterodimer band was obtained (130 kDa band). Due to the association between enzymatic activity and brightness of the bands (28), higher activity of both forms of MMP-2 and MMP-9 were observed in the RC fluid.

The activity of MMP-2 and MMP-9 in the cyst capsule is shown in Fig. 2. The activity of different MMP forms were measured as described above. Of note, higher activity of pro-MMP-9 $(\sim 92 \mathrm{kDa})$ in the RC capsule was observed.

In the present study, significantly increased activity of MMP-9 was observed in the RC fluid compared with the

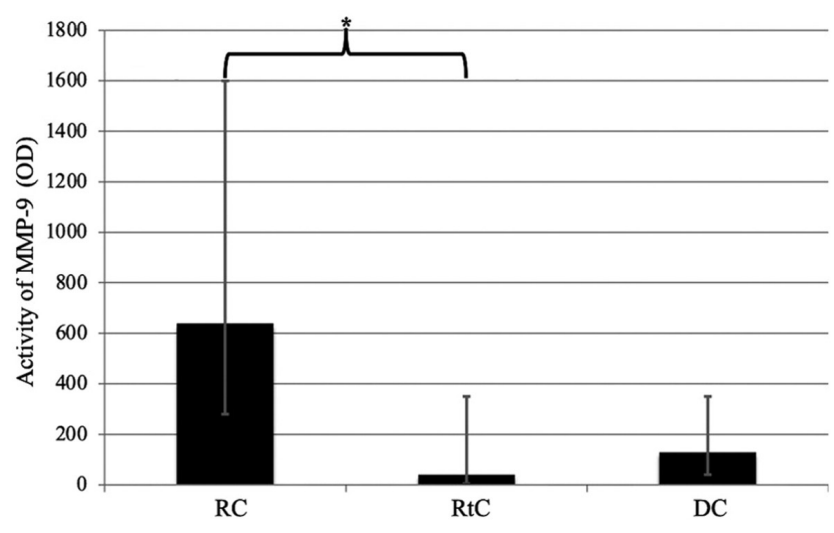

Figure 3. Activity of MMP-9 in the cyst fluid from patients diagnosed with RC, DC or RtC. Statistically significantly higher activity of MMP-9 was observed in the RCs compared with the RtCs. ${ }^{*} \mathrm{P}=0.0065$. OD, optical density; $\mathrm{RC}$, radicular cyst; RtC, retention cyst; DC, dentigerous cyst; MMP, matrix metalloproteinase.

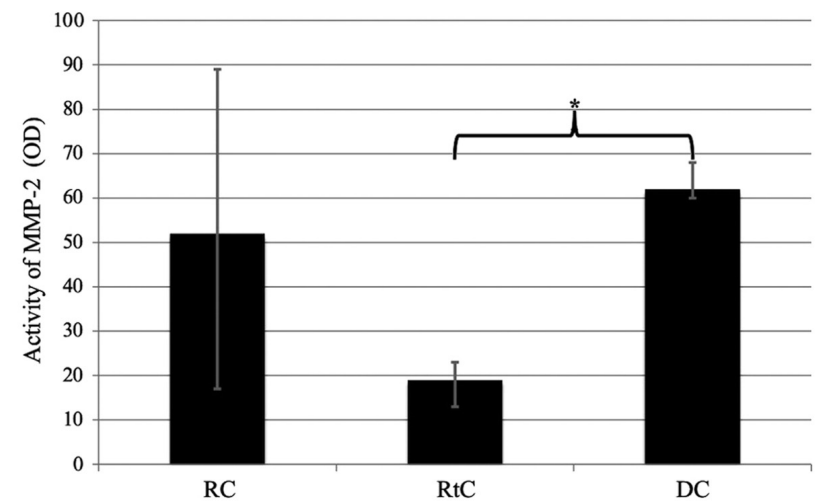

Figure 4. Activity of MMP-2 in the cyst capsules of patients diagnosed with $\mathrm{RC}, \mathrm{DC}$ or RtC. The difference between RtC and DC were statistically significant. ${ }^{*} \mathrm{P}=0.046$, Kruskal-Wallis; $\mathrm{P}<0.05$, post hoc Dunns' test. OD, optical density; RC, radicular cyst; RtC, retention cyst; DC, dentigerous cyst; MMP, matrix metalloproteinase.

RtC fluid ( $\mathrm{P}=0.0065$; Fig. 3). No significant differences were observed between DGs and other types of lesions. Moreover, decreased activity levels of MMP-2 were observed in the cyst capsule of patients with RtCs, but this difference was statistically significant only when compared with DCs ( $\mathrm{P}=0.046$; Fig. 4). 


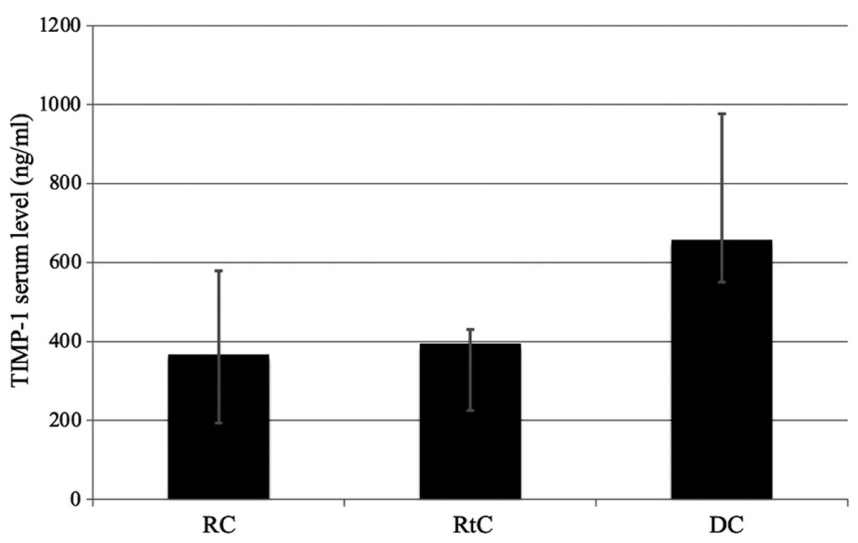

Figure 5. TIMP-1 serum levels in patients diagnosed with RC, DC or RtC. There were no significant differences in the levels of TIMP-1 between the different types of cysts. RC, radicular cyst; RtC, retention cyst; DC, dentigerous cyst; TIMP, tissue inhibitor of metalloproteinase.

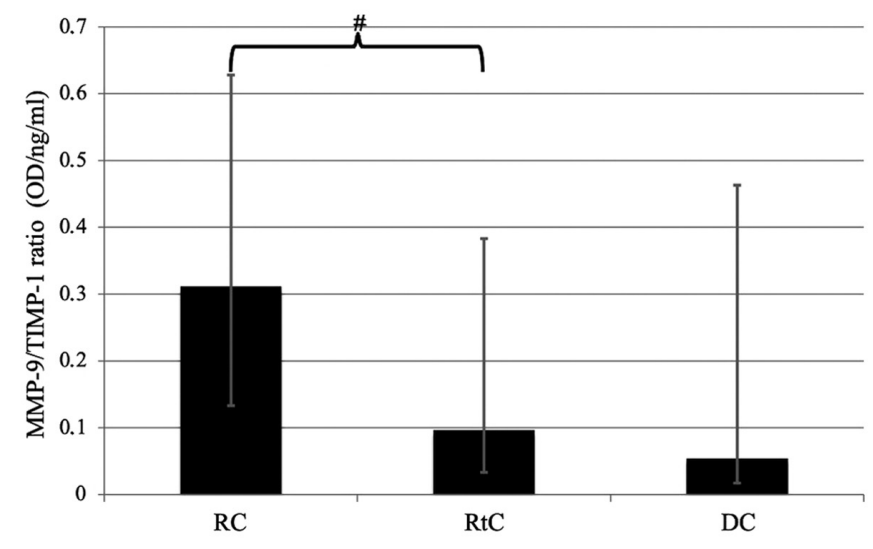

Figure 6. MMP-9/TIMP-1 ratio in the cyst fluid of patients diagnosed with $\mathrm{RC}, \mathrm{DC}$ or RtC. Comparison of the ratio in the MMP-9/TIMP-1 between $\mathrm{RC}$ and $\mathrm{RtC}$ trended towards statistical significance $(\mathrm{P}=0.09$, Kruskal-Wallis test; ${ }^{\prime} \mathrm{P}<0.05$, post hoc Dunns' test. OD, optical density; RC, radicular cyst; $\mathrm{RtC}$, retention cyst; DC, dentigerous cyst; TIMP, tissue inhibitor of metalloproteinase; MMP, matrix metalloproteinase.

Of note, MMP-9 was the most frequently detected MMP in the RC capsule group (65\% of cases). No MMP-9 was detected in any of the patients with DCs.

The TIMP-1 serum levels in patients with RCs were reduced compared with DCs and RtCs, but the difference was not statistically significant (Fig. 5). Of note, the MMP-9/TIMP-1 ratio was higher in the fluid from RCs compared with RtCs and DCs. However, the difference between the value of the ratio in RCs and RtCs only trended towards statistical significance $(\mathrm{P}=0.09$; Fig. 6).

\section{Discussion}

The biological factors that affect the development of odontogenic cysts have yet to be clearly determined (29). Due to fact that MMPs are able to degrade a wide range of substrates within the ECM, they represent one of the most common subjects of investigation in the context of elucidating the formation and expansion of cystic lesions localized in the maxillofacial region. However, current literature comparing their activity in various components of the maxillofacial cysts, as well as between different types of cysts, are limited. The comparisons of MMP activity in the cysts and in other odontogenic tumors is understandable, given their more aggressive potential. Ameloblastoma, which is described as a benign, locally aggressive odontogenic tumor, and odontogenic keratocyst $(\mathrm{OKC})$ are characterized by higher destructive potentials and a tendency to recur when compared with RCs and DCs (7). According to the World Health Organizations recommendations on the reclassification of keratocystic odontogenic tumors (KCOTs) into the group of OKCs, all references to $\mathrm{KCOT}$ in the present study should be considered as an OKC (30). It is necessary to underline that the new classification system does not modify their clinical and/or pathological status (30). Henriques et al (8) reported significantly higher MMP-9 expression in ameloblastoma and KCOTs compared with that in RCs and DCs. Furthermore, DCs exhibited slightly higher expression of this protease compared with RCs. It should be noted that the present study did not identify MMP-9 activity in the DC capsule, whereas its activity was detected in $\mathrm{RC}$ and RtC. Due to the small number of patients diagnosed with DC, further studies are necessary in order to draw more definitive conclusions. Moreover, immunohistochemical analysis demonstrated higher expression of MMP-9 within the epithelial, fibrous and vascular components in OKCs compared with DCs and RCs. Therefore, it was concluded that the levels of expression of MMP-9 may be associated with the more destructive potential of OKCs compared with that of other odontogenic cysts (31). An identical association was observed between MMP-2 activity and KCOT. Numerous studies have emphasized the involvement of MMP-2 and other enzymes from the matrixins group in the process of bone resorption and odontogenic lesion expansion. However, its activity also significantly affects KCOT development (32-34). A genetic study has also shed some light on the aforementioned conclusions. It was shown that MMP-2 gene polymorphisms may be involved in the increased aggressiveness of OKCs (29).

Comprehensive assessment of bone tissue homeostasis and the dynamics of the bone degradation process requires juxtaposition of both matrixins and their specific tissue inhibitors. A previous study confirmed increased expression of both gelatinases, TIMP-1 and TIMP-2, in ameloblastoma tissues (35). Moreover, Pinheiro et al (36) reported increased activity of MMP-1, MMP-2 and MMP-9 in particular tumor types. These enzymes, in addition to their effect on lesion expansion, may underlie the etiology behind local aggressiveness. In their view, digestion of ECM results in the release of mitogenic factors that increase the rate of tumor cell proliferation. Moreover, disturbances in the expression of the genes encoding both gelatinases may be associated with the destructive nature of ameloblastomas (29). The comparison of the expression of matrixins with that of their inhibitors and inducers may provide further insight with regard to the significance of these enzymes in the alterations of bone tissue adjacent to the pathological lesions. Expression of numerous MMPs was confirmed in the parenchymal and stromal component of calcifying odontogenic cysts (COCs) (37). Previously, a $\mathrm{COC}$ was considered to be a calcifying cystic odontogenic tumor (30). However, Prosdócimi et al (38) noticed differences in the expression levels of MMPs, their inhibitors (TIMPs 
and the membrane-anchored glycoprotein RECK) and ECM metalloproteinase inducers in the epithelium and stroma of COCs. Their results suggested that these abnormalities in ECM homeostasis may contribute to bone degradation, and assist in the determination of the characteristics of the pathological lesions.

Development of PGs and, consequently, RCs is based on the inflammatory process within the dental pulp (39). Despite the fact that residual RCs (RRCs) are characterized by an identical etiology as both PGs and RCs , the inflammatory factor contributing to the expansion of the lesion is eliminated by extraction of the affected tooth (40). Thus, despite a comparable histopathological architecture, the biochemical status of the two lesions may be completely distinct (41). It has been shown that MMP-9 is expressed in both inflammatory cysts; however, a higher level of expression was observed in RCs compared with that in RRCs (9). This correlation may suggest that RRCs have a reduced ability to degrade ECM compared with RCs. Furthermore, it is noteworthy that RCs were the only typical inflammatory lesions in the present study. The results of the present study demonstrated a statistically significantly higher gelatinolytic activity of MMP-9 in $\mathrm{RC}$ fluid and its capsule compared with that in both DG and RtC. Conversely, none of the tested samples in the group of patients diagnosed with RC exhibited prominent activity of MMP-2 compared with the other cysts. This may suggest a higher effectiveness of MMP-9 in ECM degradation in inflammatory lesions compared with MMP-2. Previous studies confirmed the involvement of MMP-9 in the etiology of other inflammatory processes $(42,43)$. Due to the similarities in the substrates between MMP-9 and MMP-2, the lack of statistically significant differences between MMP-2 activity in the serum and cyst fluid does not exclude the importance of MMP-2 in the remodeling process of ECM. However, further research is required to elucidate this association. Regarding the present study, research conducted by Teronen et al (44) also reported significant results. Activity of enzymes with a molecular weight of $92 \mathrm{kDa}$ (corresponding to pro-MMP-9) and $72 \mathrm{kDa}$ (corresponding to pro-MMP-2) and their tissue inhibitors (TIMP-1 and TIMP-2) was detected in the fluid and capsule of all odontogenic lesions (RC, RRC, DG and OKC). However, the dominant gelatinolytic activity in the cyst capsule belonged to MMP-9. It was noted that ECM degradation is possible due to partial blocking of MMPs by endogenous inhibitors. Moreover, they suggested that gelatinases may be involved in the final stages of this process, which was initiated by the activity of collagenases (MMP-1 and MMP-8) (44).

There is currently a lack of sufficient information on the role of MMPs in RtC development. To the best of our knowledge, there is only one study that has investigated MMP expression within the RtC and maxillary sinusitis (MS) fluid, which demonstrated distinct differences between the two disorders. The expression of MMP-1, -2, -3, -9 and -10 were found to be higher in the patients with MS compared with that in RtC (24). There are currently no available studies regarding the activity of matrixins in the RtC capsule. Moreover, no studies have been performed comparing MMP activity in the components of RtC and odontogenic lesions. This is particularly important due to the fact that the aforementioned lesions are characterized by diametrically different etiopathogeneses, growth dynamics and aggressiveness. In the present study, lower MMP-2 activity was observed in the RtC capsule compared with both odontogenic cysts. However, this result was statistically significant in relation only to DC. The emergence and expansion of both RCs and DCs is associated with bone tissue destruction. By contrast, RtC does not completely fill the cavity of the maxillary sinus, and no bone damage or bone remodeling of the maxillary sinus were observed (23). Upon comparing the reduced activity of MMP-2 in the RtC wall with its clinical characteristics, it may be inferred that MMP-2 serves an important role in the ECM degradation process resulting in bone destruction. Therefore, it may be considered a specific biomarker that may be used to reflect the tendency of the lesions to cause bone remodeling, with potential implications in treatment planning or monitoring of outcomes. However, this hypothesis requires further investigations with larger cohorts. Other reports have focused on the pathologies of the paranasal sinuses and nasal cavity, for example, nasal polyps (NP) and chronic inflammation of the sinuses and/or nasal cavity. Literature data have reported increased expression of MMP-9 and TIMP-1 in NP and chronic sinusitis compared with unaffected sinus mucosa of a control group. This suggests the involvement of MMP-9 in the pathogenesis of these conditions $(45,46)$.

As gelatinases are inhibited in vivo by their specific tissue inhibitors, the MMP-9/TIMP-1 and MMP-2/TIMP-2 ratios were compared in serum and fluids collected from various types of cysts. The MMP-9/TIMP-1 ratio was only slightly increased in RC fluid compared with RtC, and this increase trended towards statistical significance. The obtained results may confirm the role of MMP-9 in the pathogenesis of this type of cyst. Of note, the analyzed ratios were based on the inhibitor with the highest affinity for a given enzyme. However, each of the MMPs can also be inhibited by other tissue inhibitors, as well as by non-specific protease inhibitors $(47,48)$. Therefore, conclusions on the actual in vivo activity of MMPs should be drawn with caution.

It is worth noting that, in several previous studies, immunohistochemical analysis, ELISA or western blotting have been used $(8,9,42,44)$. Due to the characteristics of these methods, it is not possible to differentiate between the forms (active, latent or dimer) of specific matrixins. The results obtained in the present study also provide information regarding the activity of latent forms of MMPs, which are unable to perform proteolysis in vivo. ELISA and western blot analysis cannot evaluate the catalytic ability of MMPs, and do not reflect the dynamics of processes occurring within the ECM. The use of gelatin zymography provides additional information, as it also analyzes the latent, inactive in vivo forms of gelatinases. Matrixin proforms are activated by SDS as a component of the zymographic gel. In this context, it was possible to observe the enzymatic activity of pro-MMP-2 and pro-MMP-9, as shown on representative zymograms. Another advantage of zymography is also the ability to detect MMPs at low concentrations (pg/ $/ \mu \mathrm{l})$ (49). However, expressing their activity in OD units only indirectly reflects the concentration of these enzymes. It appears that the use of gelatin zymography more accurately reflects the dynamics of processes occurring between the ECM and MMPs.

The findings of the present study confirmed the involvement of MMPs in the development of cystic lesions within the 
maxillofacial region. Despite the promising results, certain issues require further study to resolve. More detailed research on RtCs is necessary, as the current state of knowledge on these lesions is considerably limited. Further study may provide novel insight into the nature of MMPs, and may also improve the accuracy of the complicated differential diagnosis of benign, non-odontogenic cystic lesions of the maxillary sinuses. The juxtaposition of benign cystic lesion with pathologies characterized by a more aggressive nature may provide further valuable conclusions. Moreover, the combination of gelatin zymography with other methods, such as immunohistochemical assays, may allow for identification of the cellular components of the lesions that are primarily responsible for the expression of MMPs.

In conclusion, MMP-9 is involved in the pathogenesis of $\mathrm{RC}$, based on the observation that it exhibited the highest degree of activity in the fluids collected from the cyst cavity when compared with the other studied lesions. It should also be emphasized that the presence of this gelatinase was confirmed in RC walls, and the proportion of expression of MMP-9 was highest amongst all lesions. Conversely, the low activity of MMP-2 in the RtC walls suggests a limited effect of this gelatinase on the development of this type of lesion. Thus, both gelatinases may serve a potential role in the differential clinical diagnosis of craniofacial cysts and may serve as supplementary elements in histopathological studies. Furthermore, it appears that MMP-9 may be of value as a specific biomarker in RC etiology. However, due to the aforementioned limitations, further, more complex investigations are required to draw definitive conclusions.

\section{Acknowledgements}

Not applicable.

\section{Funding}

The present study was funded by the Medical University of Lublin.

\section{Availability of data and materials}

The datasets used and/or analyzed during the present study are available from the corresponding author on reasonable request.

\section{Authors' contributions}

KK wrote the manuscript and performed the experiments. DLK performed the experiments, and wrote and reviewed the manuscript. TT supervised the study, interpreted the data, and wrote and revised the manuscript. JK conceived the study, wrote and revised the manuscript, and analyzed the data. All authors read and approved the final manuscript. KK and JK confirm the authenticity of all the raw data.

\section{Ethics approval and consent to participate.}

The present study was approved by the Ethical Committee at the Medical University of Lublin (approval no. KE-0254/5/2017). Each patient recruited provided singed informed consent. All procedures were performed in accordance with the guidelines described in the Declaration of Helsinki.

\section{Patient consent for publication}

Not applicable.

\section{Competing interests.}

The authors declare that they have no competing interests.

\section{References}

1. Koivisto T, Bowles WR and Rohrer M: Frequency and distribution of radiolucent jaw lesions: A retrospective analysis of 9,723 cases. J Endod 38: 729-732, 2012.

2. Lawal A, Adisa A and Sigbeku O: Cysts of the oro-facial region: A Nigerian experience. J Oral Maxillofac Pathol 16: 167-171, 2012.

3. Johnson NR, Gannon OM, Savage NW and Batstone MD: Frequency of odontogenic cysts and tumors: A systematic review. J Investig Clin Dent 5: 9-14, 2014.

4. Kadam NS, Ataide IN, Raghava P, Fernandes M and Hede R: Management of large radicular cyst by conservative surgical approach: A case report. J Clin Diagn Res 8: 239-241, 2014.

5. Tortorici S, Amodio E, Massenti MF, Buzzanca ML, Burruano F and Vitale F: Prevalence and distribution of odontogenic cysts in Sicily: 1986-2005. J Oral Sci 50: 15-18, 2008.

6. Rajendra Santosh AB: Odontogenic Cysts. Dent Clin North Am 64: 105-119, 2020

7. Ali A, Asif M, Ahmad B, Jamal S, Ali I and Khadim MT: Stromal Expression of CD10 by immunohistochemistry in odontogenic keratocyst (OKC), dentigerous and radicular cysts and its correlation with local recurrence and aggressive behaviour. Asian Pac J Cancer Prev 20: 249-253, 2019.

8. Henriques ÁC, Vasconcelos MG, Galvão HC, de Souza LB and de Almeida Freitas R: Comparative analysis of the immunohistochemical expression of collagen IV, MMP-9, and TIMP-2 in odontogenic cysts and tumors. Oral Surg Oral Med Oral Pathol Oral Radiol Endod 112: 468-475, 2011.

9. Ruiz PA, Toledo OA, Nonaka CF, Pinto LP and Souza LB: Immunohistochemical expression of vascular endothelial growth factor and matrix metalloproteinase-9 in radicular and residual radicular cysts. J Appl Oral Sci 18: 613-620, 2010.

10. Liang H, Xu J, Xue M and Jackson C: Matrix metalloproteinases in bone development and pathology: Current knowledge and potential clinical utility. Metalloproteinases Med 3: 93-102, 2016.

11. Kurzepa J,Baran M, Watroba S,Barud Mand Babula D: Collagenases and gelatinases in bone healing. The focus on mandibular fractures. Curr Issues Pharm Med Sci 27: 121-126, 2014.

12. Cunningham LA, Wetzel M and Rosenberg GA: Multiple roles for MMPs and TIMPs in cerebral ischemia. Glia 50: 329-339, 2005.

13. Palosaari H, Wahlgren J, Larmas M, Rönkä H, Sorsa T, Salo T and Tjäderhane L: The expression of MMP-8 in human odontoblasts and dental pulp cells is down-regulated by TGF-beta1. J Dent Res 79: 77-84, 2000.

14. Shay G, Lynch CC and Fingleton B: Moving targets: Emerging roles for MMPs in cancer progression and metastasis. Matrix Biol 44-46: 200-206, 2015.

15. Pardo A and Selman M: MMP-1: The elder of the family. Int J Biochem Cell Biol 37: 283-288, 2005.

16. Moore CS and Crocker SJ: An alternate perspective on the roles of TIMPs and MMPs in pathology. Am J Pathol 180: 12-16, 2012.

17. Descamps FJ, Martens E and Opdenakker G: Analysis of gelatinases in complex biological fluids and tissue extracts. Lab Invest 82: 1607-1608, 2002

18. Hannocks M-J, Zhang X, Gerwien H, Chashchina A, Burmeister M, Korpos E, Song J and Sorokin L: The gelatinases, MMP-2 and MMP-9, as fine tuners of neuroinflammatory processes. Matrix Biol 75-76: 102-113, 2019.

19. Pereira Faustino IS, Azevedo RS and Takahama A Jr: Metalloproteinases 2 and 9 immunoexpression in periapical lesions from primary endodontic infection: Possible relationship with the histopathological diagnosis and the presence of pain. J Endod 42: 547-551, 2016. 
20. Hadziabdic N, Kurtovic-Kozaric A, Pojskic N, Sulejmanagic N and Todorovic L: Gene-expression analysis of matrix metalloproteinases 1 and 2 and their tissue inhibitors in chronic periapical inflammatory lesions. J oral Pathol Med Off Publ Int Assoc Oral Pathol Am Acad Oral Pathol 45: 224-230, 2016.

21. Bariş E, Sengüven B, Bozkaya S and Oygür T: Immunohistochemical analysis of matrix metalloproteinases-1,-9 and tenascin in odontogenic lesions. Eur J Inflamm 12: 419-427, 2014

22. Suojanen J, Lehtonen N, Färkkilä E, Hietanen J, Teronen O, Sorsa $\mathrm{T}$ and Hagström J: Common matrix metalloproteinases (MMP-8, -9, -25, and -26) cannot explain dentigerous cyst expansion. J Clin Diagn Res 8: ZC82-ZC85, 2014.

23. Bal M, Berkiten G and Uyanık E: Mucous retention cysts of the paranasal sinuses. Hippokratia 18: 379, 2014.

24. Kim SM, Eo MY, Cho YJ, Kim YS and Lee SK: Differential protein expression in the secretory fluids of maxillary sinusitis and maxillary retention cyst. Eur Arch Otorhinolaryngol 274: 215-222, 2017.

25. Giotakis EI and Weber RK: Cysts of the maxillary sinus: A literature review. Int Forum Allergy Rhinol 3: 766-771, 2013

26. World Medical Association Declaration of Helsinki: Ethical Principles for Medical Research Involving Human Subjects 2018.

27. Babula D, Kocot J, Horecka A, Baran M and Kurzepa J: Different patterns of gelatinolytic activity in pituitary macro- and microadenomas. Clin Neurol Neurosurg 158: 90-92, 2017.

28. Vandooren J, Geurts N, Martens E, Van den Steen PE and Opdenakker G: Zymography methods for visualizing hydrolytic enzymes. Nat Methods 10: 211-220, 2013

29. Aloka D, Padmakumar SK, Sathyan S, Sebastian M, Banerjee M and Beena VT: Association of matrix metalloproteinase 2 and matrix metalloproteinase 9 gene polymorphism in aggressive and nonaggressive odontogenic lesions: A pilot study. J Oral Maxillofac Pathol 23: 158, 2019.

30. Kaczmarzyk T, Stypułkowska J and Tomaszewska R: Update of the WHO classification of odontogenic and maxillofacial bone tumours. J Stomatol (Brux) 70: 484-506, 2017.

31. de Andrade Santos PP, de Aquino ARL, Oliveira Barreto A, de Almeida Freitas R, Galvão HC and de Souza LB: Immunohistochemical expression of nuclear factor $\kappa \mathrm{B}$, matrix metalloproteinase 9, and endoglin (CD105) in odontogenic keratocysts, dentigerous cysts, and radicular cysts. Oral Surg Ora Med Oral Pathol Oral Radiol Endod 112: 476-483, 2011.

32. Amm HM, Casimir MD, Clark DB, Sohn P and MacDougall M: Matrix metalloproteinase expression in keratocystic odontogenic tumors and primary cells. Connect Tissue Res 55 (Suppl 1): 97-101, 2014.

33. Scariot R, Morosini IC, Torres-Pereira CC, Amenabar JM, Rebellato NL and Gugisch RC: Immunohistochemical analysis of metalloproteases in dentigerous cysts, radicular cysts and keratocystic odontogenic tumors: Systematic review. Stomatos 18: 34 2012.

34. Nadalin MR, Fregnani ER, Silva-Sousa YTC and da Cruz Perez DE: Presence of myofibroblasts and matrix metalloproteinase 2 in radicular cysts, dentigerous cysts, and keratocystic odontogenic tumors: A comparative immunohistochemical study. J Endod 38: 1363-1367, 2012.

35. Kumamoto $H$, Yamauchi $K$, Yoshida $M$ and Ooya $K$ : Immunohistochemical detection of matrix metalloproteinases (MMPs) and tissue inhibitors of metalloproteinases (TIMPs) in ameloblastomas. J Oral Pathol Med 32: 114-120, 2003.
36. Pinheiro JJ, Freitas VM, Moretti AI, Jorge AG and Jaeger RG: Local invasiveness of ameloblastoma. Role played by matrix metalloproteinases and proliferative activity. Histopathology 45: 65-72, 2004.

37. Ribeiro BF, Ferreira de Araújo CR, dos Santos BR and de Almeida Freitas R: Immunohistochemical expression of matrix metalloproteinases $1,2,7,9$, and 26 in the calcifying cystic odontogenic tumor. Oral Surg Oral Med Oral Pathol Oral Radiol Endod 112: 609-615, 2011.

38. Prosdócimi FC, Rodini CO, Sogayar MC, Sousa SC, Xavier FC and Paiva KB: Calcifying Cystic Odontogenic Tumour: Immunohistochemical expression of matrix metalloproteinases, their inhibitors (TIMPs and RECK) and inducer (EMMPRIN). J Oral Pathol Med 43: 545-553, 2014.

39. de Paula-Silva FW, D'Silva NJ, da Silva LA and Kapila YL: High matrix metalloproteinase activity is a hallmark of periapical granulomas. J Endod 35: 1234-1242, 2009.

40. Bava FA, Umar D, Bahseer B and Baroudi K: Bilateral radicular cyst in mandible: an unusual case report. J Int Oral Health 7: 61-63, 2015

41. Muglali M, Komerik N, Bulut E, Yarim GF, Celebi N and Sumer M: Cytokine and chemokine levels in radicular and residual cyst fluids. J Oral Pathol Med 37: 185-189, 2008.

42. Sheen P, O'Kane CM, Chaudhary K, Tovar M, Santillan C, Sosa J, Caviedes L, Gilman RH, Stamp G and Friedland JS: High MMP-9 activity characterises pleural tuberculosis correlating with granuloma formation. Eur Respir J 33: 134-141, 2009.

43. Zhang P, Wu C, Huang XH, Shen CL, Li L, Zhang W and Yao CZ: Aspirin suppresses TNF- $\alpha$-induced MMP-9 expression via NF- $\mathrm{KB}$ and MAPK signaling pathways in RAW264.7 cells. Exp Ther Med 14: 5597-5604, 2017.

44. Teronen O, Salo T, Konttinen YT, Rifkin B, Vernillo A, Ramamurthy NS, Kjeldsen L, Borregaard N, Hietanen J and Sorsa T: Identification and characterization of gelatinases/type IV collagenases in jaw cysts. J Oral Pathol Med 24: 78-84, 1995.

45. Lechapt-Zalcman E, Coste A, d'Ortho MP, Frisdal E, Harf A, Lafuma $C$ and Escudier E: Increased expression of matrix metalloproteinase-9 in nasal polyps. J Pathol 193: 233-241, 2001.

46. Watelet JB, Bachert C, Claeys C and Van Cauwenberge P: Matrix metalloproteinases MMP-7, MMP-9 and their tissue inhibitor TIMP-1: Expression in chronic sinusitis vs. nasal polyposis. Allergy 59: 54-60, 2004.

47. Belotti D, Paganoni P and Giavazzi R: MMP inhibitors: Experimental and clinical studies. Int J Biol Markers 14: 232-238, 1999.

48. Bourboulia D and Stetler-Stevenson WG: Matrix metalloproteinases (MMPs) and tissue inhibitors of metalloproteinases (TIMPs): Positive and negative regulators in tumor cell adhesion. Semin Cancer Biol 20: 161-168, 2010.

49. Gogly B, Groult N, Hornebeck W, Godeau G and Pellat B: Collagen zymography as a sensitive and specific technique for the determination of subpicogram levels of interstitial collagenase. Anal Biochem 255: 211-216, 1998.

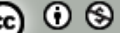

This work is licensed under a Creative Commons Attribution-NonCommercial 4.0 International (CC BY-NC 4.0) License. 